\title{
Perdão e Satisfação Conjugal: Uma Revisão Sistemática
}

\author{
Ana Isabel Mendes-Teixeira ${ }^{1}$ \\ ${ }^{1}$ Universidade do Porto, Portugal.
}

\author{
Cidália Duarte ${ }^{1}$ \\ ${ }^{1}$ Universidade do Porto, Portugal.
}

\begin{abstract}
Resumo: O objetivo deste estudo foi realizar uma revisão sistemática da literatura sobre o perdão, quando estudado, com a satisfação conjugal, possibilitando uma melhor integração empírica da investigação sobre os dois construtos. Apesar da interdisciplinaridade emergente, a investigação face a esses dois construtos têm permanecido inconsistente, existindo várias dúvidas quanto à relação entre ambos. Foi realizada uma pesquisa em diferentes bases de dados internacionais (Academic Search Complete, PsycArticles, PsycInfo, PsycCritiques, Psychology and Behavioral Sciences Collection), consultadas até 1990, retrospectivamente, permitindo uma identificação inicial de 516 estudos. Com base em critérios de inclusão e exclusão rigorosa e previamente definidos, 25 estudos, publicados entre 2002 e 2016, foram selecionados para uma análise crítica. Os resultados indicam que a maioria dos estudos aposta numa conceptualização multidimensional do perdão, emergindo a presença de uma dimensão motivacional positiva (benevolência) e a renúncia a um estado motivacional negativo (vingança, evitamento, retaliação). Os dados sugerem relações positivas entre perdão e satisfação conjugal: o perdão promove níveis superiores de satisfação conjugal; níveis superiores de satisfação conjugal promovem um maior perdão; a relação entre os construtos pode ser bidirecional. Contudo, a maioria dos estudos incluídos revela um design metodológico transversal, limitando a exploração e a retirada de conclusões acerca da direção das relações causais entre os dois construtos. Revela-se fundamental o desenvolvimento de estudos futuros com designs metodológicos mais complexos, permitindo uma compreensão mais rigorosa e o alargamento do campo de estudos.
\end{abstract}

Palavras-chave: Perdão, Satisfação conjugal, Conjugalidade, Revisão sistemática.

\section{Forgiveness and Marital Satisfaction: A Systematic Review}

\begin{abstract}
This study aimed to conduct a systematic review on forgiveness associated with marital satisfaction, enabling a better empirical integration of the literature on them. Despite the emergent interdisciplinarity, the true association between these constructs still raise many doubts, indicating the inconsistency in studies on this topic. Thus, this study conducted a retrospective search for articles published up from 1900 in the Academic Search Complete, PsycArticles, PsycInfo, PsycCritiques, Psychology, and Behavioral Sciences Collection databases, identifying 516 studies. After applying strict predefined inclusion and exclusion criteria, 25 studies published between 2002 and 2016 were selected for a critical analysis. The results indicate that most studies rely on a multidimensional conceptualization of forgiveness, resulting in a positive motivational dimension (benevolence) and the renouncement to a negative motivational state (revenge, avoidance or retaliation). Forgiveness and marital satisfaction were also positively and bidirectionally associated, with forgiveness promoting higher levels of marital satisfaction and higher levels of marital satisfaction promoting greater forgiveness. However, most of the studies adopted a cross-sectional design, limiting exploration and conclusions regarding the direction of causal relationships between the two constructs. Further studies on the theme, with more complex methodological designs, are essential for a more rigorous understanding and the broadening of the field of studies.
\end{abstract}

Keywords: Forgiveness, Marital satisfaction, Conjugality, Systematic review. 


\title{
Perdón y Satisfacción Conyugal: Una Revisión Sistemática
}

\begin{abstract}
Resumen: El objetivo de este estudio fue realizar una revisión sistemática de la literatura acerca del perdón asociado con la satisfacción conyugal, posibilitando una mejor integración empírica de la investigación sobre los dos constructos. A pesar de la emergente interdisciplinaridad, es inconsistente la investigación con estos dos constructos, lo que plantea dudas sobre la relación entre ambos. Se realizó una búsqueda en diferentes bases de datos internacionales (Academic Search Complete, PsycArticles, PsycInfo, PsycCritiques, Psychology and Behavioral Sciences Collection), consultadas hasta 1990 (retrospectivamente), y se obtuvo inicialmente 516 estudios. Con base en criterios de inclusión y exclusión rigurosos y previamente definidos, se seleccionaron 25 estudios, publicados entre 2002 y 2016, para el análisis crítico. Los resultados indican que la mayoría de los estudios presentan una conceptualización multidimensional del perdón, emergiendo la presencia de una dimensión motivacional positiva (benevolencia) y la renuncia a un estado motivacional negativo (venganza, evitación, represalia). Los datos evidencian relaciones positivas entre perdón y satisfacción conyugal: el perdón promueve niveles superiores de satisfacción conyugal; los altos niveles de satisfacción conyugal favorecen más el perdón; la relación entre los constructos puede ser bidireccional. Sin embargo, la mayoría de los estudios incluidos revelan un diseño metodológico transversal, limitando la exploración y la retirada de conclusiones acerca de la dirección de las relaciones causales entre los dos constructos. Es fundamental desarrollar estudios futuros con diseños metodológicos más complejos para obtener una comprensión más rigurosa y la ampliación del campo de estudios.
\end{abstract}

Palabras clave: Perdón, Satisfacción conyugal, Conyugalidad, Revisión sistemática.

\section{Introdução}

Paradoxalmente, são aqueles quem mais amamos que mais, provavelmente, ofendemos (Fincham $\&$ Beach, 2002). Para lidar com tais situações, o perdão poderá ser um caminho a percorrer, reconhecido como capaz de promover o bem-estar físico e psicológico (McCullough, 1997), contribuindo para a satisfação com a vida em geral (Karremans, Van Lange, Ouwerkerk, \& Kluwer, 2003). Na prática clínica, emergem cada vez mais intervenções que promovem o perdão, tanto no âmbito da terapia familiar quanto no da terapia de casal (Murray, 2002; Reed \& Enright, 2006), o que denota, uma vez mais, os contornos positivos.

Uma das grandes questões que caracteriza o estudo do perdão está relacionada com a definição, constatando-se que a investigação tem destacado a importância de se considerar a natureza multidimensional desse construto (Enright \& The Human Development Study Group, 1991), que pode ser definido como como um conjunto de mudanças motivacionais que levam à inibição de possíveis respostas negativas, como a vingança, a retaliação ou o distanciamento, e ao aumento de respostas positivas, como a benevolência face ao ofensor (Guzmán-González, Alfaro, \& Armenta, 2013). Distinguir essas duas dimensões é importante, pois elas podem ter diferentes determinantes, correlações e consequências (Fincham \& Beach, 2002), além de que, no contexto dos relacionamentos românticos, essas dimensões parecem operar de modo distinto em cada um dos parceiros (Fincham, Hall, \& Beach, 2005).

É consensual na literatura a ideia de que o perdão é um processo em vez de um ato específico (Fincham, Hall, \& Beach, 2006), exigindo, naturalmente, seu próprio tempo e podendo ser facilitado, ou dificultado, por determinadas variáveis relacionadas com o ajustamento psicológico (Fehr, Gelfand, \& Nag, 2010) ou até variáveis demográficas, suscitando no gênero mais resultados inconclusivos à medida que estudos demonstraram não existir diferenças significativas entre homens e mulheres (Fehr et al., 2010; Pinto \& Barros-Oliveira, 2006; Rique \& Camino, 2009; Taysi, 2010), ou outros que revelaram que as mulheres são mais propensas a perdoar do que os homens (Finkel, Rusbult, Kumashiro, \& Hannon, 2002; Miller, Worthington Jr., \& McDaniel, 2008). Essa diferença poderá ser explicada pelo fato de o sexo feminino 
estar frequentemente associado a uma maior gentileza, empatia e orientação para os relacionamentos, além de maior autorrevelação de sentimentos (Hook, Gernstein, Detterich, \& Gridley, 2003). Comparativamente, o sexo masculino parece estar associado a níveis superiores de raiva e retaliação, a uma menor exibição de comportamentos íntimos e uma maior tendência para a retirada em situações de conflito (Prager \& Fincham, 2001).

No sentido de compreender que possíveis variáveis são suscetíveis de influenciar o processo de perdão, McCullough et al. (1998) elaboraram um modelo de quatro categorias de determinantes do perdão bastante influente na literatura. De acordo com o modelo, os determinantes sociocognitivos, relacionados com a forma como o parceiro ofendido pensa e sente em relação à ofensa e ao ofensor (empatia, ruminação e atribuições causais), representam os determinantes mais proximais do perdão. Em relação aos determinantes relacionados com a natureza da ofensa (severidade da ofensa e pedido de desculpa), eles tendem a moldar indiretamente o perdão por meio de variáveis sociocognitivas (McCullough, 1997; McCullough et al., 1998). Já os determinantes relacionados às características de personalidade da pessoa ofendida (afabilidade e neuroticismo) parecem ser os determinantes menos proximais do perdão (McCullough et al., 1998). Por fim, estão as qualidades do relacionamento interpessoal, em que o perdão tem lugar (McCullough et al., 1998), destacando-se variáveis como o compromisso, a intimidade e a satisfação conjugal, construto sob o qual recai a nossa atenção, estando bem estabelecido na literatura seu valor fundamental na vida de um casal (Scorsolini-Comin \& Santos, 2008), associada a sentimentos de felicidade na vida em geral (Hernandez \& Oliveira, 2003).

Com efeito, a satisfação conjugal pode ser definida como a avaliação subjetiva e pessoal que cada parceiro faz sobre a relação conjugal (Thompson, 1998). Sendo igualmente um construto complexo e multidimensional (Mosmann, Wagner, \& Féres-Carneiro, 2006), sofre a influência de diversos fatores (Norgren, Souza, Kaslow, Hammerschmidt, \& Sharlin, 2004), nomeadamente, dos processos afetivos (amor, intimidade e compromisso), cognitivos (expectativas) e comportamentais (comunicação e conflitos) que ocorrem durante as interações conjugais (Halford \& Moore, 2002), das características individuais dos parceiros (traços de personalidade e padrões de vinculação), das variáveis demográficas (sexo), contextuais (família de origem) e das transições normativas e não- -normativas ao longo do ciclo de vida familiar (Olson, 2000; Sharlin, Kaslow, \& Hammerschmidt, 2000; Wagner \& Falcke, 2001).

Mas que ingredientes possuem os casais satisfeitos que os distingue dos insatisfeitos? Segundo Gottman e Silver (2001), os primeiros se pautam por uma dinâmica em que os pensamentos, sentimentos e comportamentos positivos prevalecem sobre os negativos, sendo classificados como casais emocionalmente felizes. Dada a importância da satisfação conjugal para a saúde física e emocional, a literatura tem procurado continuamente identificar que fatores contribuem para essa satisfação (Guzmán-González et al., 2013). Por meio de um estudo envolvendo parceiros em casamento há mais de vinte anos, Fenell (1993) reuniu dez características para um casamento saudável e satisfatório, sendo que, entre elas, constava a vontade de perdoar e ser perdoado.

Inevitavelmente, no seio das relações românticas os parceiros tendem a ser, simultaneamente, ou não, autores e vítimas de ofensas (Leary, Springer, Negel, Ansell, \& Evans, 1998). Embora o perdão não implique necessariamente a reconciliação, ele está associado a resultados positivos em relacionamentos próximos (Burr, Marks, \& Day, 2012), sendo visto como um mecanismo de manutenção do relacionamento (Rusbult, Hannon, Stocker, \& Finkel 2005), oferecendo aos parceiros a oportunidade de restaurar a satisfação conjugal aos níveis da pré-ofensa, ou promover o aumento desses níveis, fortalecendo, assim, o relacionamento (Waldron \& Kelley, 2008). Portanto, parece que quanto mais os parceiros românticos perdoam, mais realizam interpretações conjugais positivas, sentem igual poder na relação (Gordon \& Baucom, 1998) e evitam, em situações de conflito, cadeias de reciprocidade negativa (Barnett \& Youngberg, 2004).

Embora se assista a uma emergência crescente na investigação que documenta uma relação positiva entre o perdão e a satisfação conjugal, até a data, o mecanismo ou os processos subjacentes à relação entre ambos os construtos permanecem por esclarecer, existindo várias questões sem respostas consistentes, nomeadamente, se a relação é causal e, em caso afirmativo, qual a direção dos possíveis efeitos (Allemand, Amberg, Zimprich, \& Fincham, 2007; Braithwaite, Selby, \& Fincham, 2011; Fincham \& Beach, 2007). Ademais, a investigação no âmbito do perdão é pautada por uma negligência da dimensão temporal (McCullough, Fincham, \& Tsang, 2003), além de carecer de integra- 
ção empírica, existindo escassas tentativas de analisar sistematicamente a grande quantidade de informação acumulada, como resultado da evolução interdisciplinar da investigação do construto (Fehr et al., 2010). O presente estudo é o primeiro de que temos conhecimento, conforme análise prévia da literatura, a se debruçar numa análise criteriosa de publicações que reportam relações entre o perdão e um determinante relacional específico, nomeadamente, a satisfação conjugal, permitindo, assim, melhor integração empírica da literatura existente sobre os dois construtos. Deste modo, nosso principal objetivo foi rever de forma sistemática a literatura do perdão quando estudado com a satisfação conjugal, por meio de uma identificação, seleção e análise criteriosa de publicações que fazem referência simultânea aos dois construtos. Para tal, foram formuladas duas questões orientadoras de pesquisa: a) determinar e examinar criticamente quais os estudos que mencionam associações entre o perdão e a satisfação conjugal em relações românticas adultas; e b) compreender quais as definições conceituais utilizadas nos estudos relativos ao perdão quando examinado com a satisfação conjugal.

\section{Método}

A identificação e posterior seleção dos artigos foram realizadas por critérios de inclusão e exclusão definidos a priori. Os critérios de inclusão foram os seguintes: a) estudos com indivíduos adultos (a partir dos 18 anos) envolvidos em relações românticas (namoro, casamento, união de fato ou recasamento) com tempo de relacionamento médio igual ou superior a dois anos - essa fase inicial parece ser caracterizada por um período de aprendizagem, adaptação, mudança e de maiores conflitos (Noller \& Feeney, 1998) - e em coabitação - a passagem para a vivência conjunta acarreta um conjunto de desafios, permitindo aos casais treinarem os papéis conjugais (Mead, 1970) - e que incluam o perdão e a satisfação conjugal; b) estudos que incluam as variáveis perdão e satisfação conjugal (ainda que estejam associadas outras variáveis psicológicas a esses dois construtos); c) publicações a partir de 1990. Com relação aos critérios de exclusão: a) estudos com indivíduos adultos envolvidos em relações românticas, com tempo de relacionamento médio inferior a dois anos de relacionamento e que não coabitem; b) publicações que não disponibilizem o full-text, c) comentários, abstracts de conferências e congressos, revisões sistemáticas, livros, teses e dissertações. Também não estão previstos constrangimentos linguísticos (à exceção da língua chinesa e árabe) nem geográficos, de modo a diminuir o risco de viés.

Face à estratégia de pesquisa, foi realizada por meio de bases de dados bibliográficas internacionais, consultadas, retrospectivamente, até o ano de 1990 e relacionadas com a psicologia: Academic Search Complete, PsycArticles, PsycInfo, PsycCritiques, Psychology and Behavioral Sciences Collection. As palavras-chave utilizadas foram as seguintes: forgiveness (perdão), marital satisfaction (satisfação conjugal), marital quality (qualidade conjugal), marriage satisfaction (satisfação no casamento), marriage (casamento), romantic relationships (relações românticas), couple (casal), dating (namoro), cohabitation (coabitação), couple satisfaction (satisfação do casal), remarriage (recasamento), relationship satisfaction (satisfação no relacionamento), que foram cruzadas utilizando o operador booleano "and".

Com o intuito de diminuir o número de artigos perdidos e o risco de viés (Higgins \& Green, 2011), a avaliação da elegibilidade de cada estudo foi realizada por dois revisores independentes. As divergências encontradas foram posteriormente debatidas de modo a se estabelecer um consenso.

\section{Resultados}

A estratégia de pesquisa utilizada permitiu a identificação de 516 estudos (ver Figura 1) nas seguintes bases: Academic Search Complete ( $n=143)$, PsycArticles $(n=33)$, PsycInfo $(n=303)$ e Psychology and Behavioral Sciences Collection $(n=37)$. Do total, 286 foram automaticamente eliminados por se tratar de duplicados e 2 foram excluídos devido a constrangimentos linguísticos. Da leitura dos títulos e palavras-chave, 166 artigos foram excluídos: 24 por não disponibilizarem o full-text, 66 por serem teses/dissertações, 2 por serem comentários, 12 por serem livros, 13 por não terem relação com as questões de investigação e 49 por as variáveis perdão e satisfação conjugal não estarem contempladas simultaneamente no estudo. Assim sendo, 62 artigos foram selecionados para leitura e análise dos respectivos resumos. Desses, 37 foram excluídos por não cumprirem os critérios de inclusão: 15 por conta de o tempo do relacionamento romântico ser inferior a dois anos, um porque os participantes não estarem envolvidos num rela- 
cionamento romântico, 8 por as variáveis perdão e satisfação conjugal não estarem contempladas simultaneamente no estudo, um porque a amostra do estudo ser constituída apenas por um participante, 2 por serem artigos de opinião, um por ser revisão de literatura e 9 por não terem relação com as questões orientadoras de investigação. Deste modo, foram incluídos na revisão 25 estudos para leitura e análise crítica. O resumo das características dos estudos incluídos encontra-se na Tabela 1.

Tabela 1

Caracterização dos estudos analisados.

\begin{tabular}{|c|c|c|c|c|c|}
\hline Id. & Título do estudo & Ano & País & $\begin{array}{c}\text { Design } \\
\text { metodológico }\end{array}$ & $\begin{array}{c}\text { Conceptualização } \\
\text { perdão }\end{array}$ \\
\hline 1 & $\begin{array}{l}\text { A "Perfect" Storm: Perfectionism, Forgiveness, and } \\
\text { Marital Satisfaction }\end{array}$ & 2011 & EUA & Transversal & - \\
\hline 2 & $\begin{array}{l}\text { A Relational Approach to Religion and Spirituality } \\
\text { in Marriage: The Role of Couples' Religious } \\
\text { Communication in Marital Satisfaction }\end{array}$ & 2015 & EUA & Transversal & - \\
\hline 3 & $\begin{array}{l}\text { An Empirical Investigation into Justice, Grace, and } \\
\text { Forgiveness: Paths to Relationship Satisfaction }\end{array}$ & 2013 & EUA & Transversal & Unidimensional \\
\hline 4 & $\begin{array}{l}\text { Dispositional Forgiveness of Self, Others, and } \\
\text { Situations }\end{array}$ & 2005 & EUA & Transversal & Multidimensional \\
\hline 5 & $\begin{array}{l}\text { Does Love Mean Never Having to Say You're Sorry? } \\
\text { Associations between Relationship Satisfaction, } \\
\text { Perceived Apology Sincerity, and Forgiveness }\end{array}$ & 2012 & EUA & Transversal & - \\
\hline 6 & $\begin{array}{l}\text { Forgive and Forget, or Forgive and Regret? } \\
\text { Whether Forgiveness Leads to Less or More } \\
\text { Offending Depends on Offender Agreeablenes }\end{array}$ & 2016 & EUA & Transversal & - \\
\hline 7 & Forgiveness and Conflict Resolution in Marriage & 2004 & $\begin{array}{l}\text { Reino } \\
\text { Unido }\end{array}$ & Transversal & Multidimensional \\
\hline 8 & $\begin{array}{l}\text { Forgiveness and Marital Quality: Precursor or } \\
\text { Consequence in Well-established Relationships? }\end{array}$ & 2007 & EUA & Longitudinal & Multidimensional \\
\hline 9 & $\begin{array}{l}\text { Forgiveness and Relationship Satisfaction: } \\
\text { Mediating Mechanisms }\end{array}$ & 2011 & EUA & $\begin{array}{l}\text { Estudo 1: } \\
\text { Transversal } \\
\text { Estudo 2: } \\
\text { Longitudinal }\end{array}$ & Multidimensional \\
\hline 10 & $\begin{array}{l}\text { Forgiveness in Marriage: The Role of Relationship } \\
\text { Quality, Attributions, and Empathy }\end{array}$ & 2002 & Itália & Transversal & Multidimensional \\
\hline 11 & $\begin{array}{l}\text { I Forgive Therefore I'm Committed: A } \\
\text { Longitudinal Examination of Commitment After a } \\
\text { Romantic Relationship Transgression }\end{array}$ & 2012 & Canadá & Longitudinal & Multidimensional \\
\hline 12 & $\begin{array}{l}\text { In the Wake of Transgressions: Examining } \\
\text { Forgiveness Communication in Personal } \\
\text { Relationships }\end{array}$ & 2011 & EUA & Transversal & - \\
\hline 13 & $\begin{array}{l}\text { Longitudinal Relations Between Forgiveness and } \\
\text { Conflict Resolution in Marriage }\end{array}$ & 2007 & EUA & Longitudinal & Multidimensional \\
\hline 14 & $\begin{array}{l}\text { Marital Quality, Forgiveness, Empathy, and } \\
\text { Rumination: A Longitudinal Analysis }\end{array}$ & 2005 & Itália & Longitudinal & Multidimensional \\
\hline
\end{tabular}




\begin{tabular}{|c|c|c|c|c|c|}
\hline Id. & Título do estudo & Ano & País & $\begin{array}{c}\text { Design } \\
\text { metodológico }\end{array}$ & $\begin{array}{c}\text { Conceptualização } \\
\text { perdão }\end{array}$ \\
\hline 15 & $\begin{array}{l}\text { Measuring Offence-Specific Forgiveness } \\
\text { in Marriage: The Marital Offence-Specific } \\
\text { Forgiveness Scale (MOFS) }\end{array}$ & 2009 & Itália & Transversal & Multidimensional \\
\hline 16 & $\begin{array}{l}\text { Measuring the Differences in Pairs' Marital } \\
\text { Forgiveness Scores: Construct Validity and Links } \\
\text { with Relationship Satisfaction }\end{array}$ & 2014 & Turquia & Transversal & Multidimensional \\
\hline 17 & $\begin{array}{l}\text { Pathways between Attachment and Marital } \\
\text { Satisfaction: The Mediating Roles of Rumination, } \\
\text { Empathy, and Forgiveness }\end{array}$ & 2014 & $\begin{array}{l}\text { Coreia } \\
\text { do Sul }\end{array}$ & Transversal & - \\
\hline 18 & Sanctity of Marriage and Marital Quality & 2014 & EUA & Transversal & Multidimensional \\
\hline 19 & $\begin{array}{l}\text { Self-forgiveness in Romantic Relationships: It } \\
\text { Matters to Both of Us }\end{array}$ & 2013 & Itália & Transversal & Multidimensional \\
\hline 20 & $\begin{array}{c}\text { Shared Religious Beliefs, Prayer, and Forgiveness } \\
\text { as Predictors of Marital Satisfaction. }\end{array}$ & 2015 & EUA & Transversal & - \\
\hline 21 & $\begin{array}{l}\text { The Effect of Forgiveness on Marital Satisfaction } \\
\text { in Relation to Marital Stability }\end{array}$ & 2006 & Bélgica & Transversal & - \\
\hline 22 & $\begin{array}{c}\text { The Role of Sincere Amends and Perceived Partner } \\
\text { Responsiveness in Forgiveness }\end{array}$ & 2012 & EUA & Transversal & Multidimensional \\
\hline 23 & $\begin{array}{l}\text { The role of trait Forgiveness and relationship } \\
\text { Satisfaction in episodic forgiveness }\end{array}$ & 2007 & Suíça & Transversal & Multidimensional \\
\hline 24 & $\begin{array}{l}\text { Widening Spheres of Impact: The Role of } \\
\text { Forgiveness in Marital and Family Functioning }\end{array}$ & 2009 & EUA & Transversal & Multidimensional \\
\hline 25 & $\begin{array}{l}\text { You Did What? The Relationship Between } \\
\text { Forgiveness Tendency, Communication of } \\
\text { Forgiveness, and Relationship Satisfaction in } \\
\text { Married and Dating Couples }\end{array}$ & 2014 & EUA & Transversal & - \\
\hline
\end{tabular}

Com relação ao ano de publicação, os estudos situam-se entre 2002 e 2016. Numa análise transcultural, verifica-se que a maioria foi realizada na América do Norte (Estados Unidos e Canadá). Quanto ao design metodológico, quatro estudos são longitudinais (Fincham \& Beach, 2007; Fincham, Beach, \& Davila, 2007; Paleari, Regalia, \& Fincham, 2005; Ysseldyk \& Wohl, 2012), um é longitudinal e transversal (Braithwaite et al., 2011) e os restantes possuem design transversal.

Considerando todos os estudos incluídos nesta revisão, o número total de participantes é de 11.831, sendo que 3.508 eram casais (isto é, ambos os membros do casal participaram, comparativamente com os restantes, em que só participou um elemento da díade). Todos os participantes tinham idades compreendidas entre os 17 e 82 anos.

Numa caracterização mais detalhada da amostra, a respeito do tempo de relacionamento dos par- ticipantes, há variação de 6 meses a 46 anos (novamente, o valor médio do tempo de relacionamento foi superior a 2 anos em todos os estudos). Em relação ao estatuto conjugal e à orientação sexual, dos estudos que particularizam essa informação, prevaleceu o casamento e a orientação predominantemente heterossexual.

Os objetivos dos estudos incluídos se revelaram mais abrangentes do que a finalidade desta revisão sistemática. No entanto, quatro categorias de objetivos podem ser identificadas: a) explorar possíveis relações entre muitas variáveis psicológicas, perdão e satisfação conjugal; b) compreender as influências do perdão no sistema familiar e na resolução de conflitos da díade; c) validar instrumentos do perdão e aprofundar questões teóricas sobre o construto; d) compreender as relações existentes entre os diferentes estilos ou estratégias de comunicação do perdão e satisfação conjugal. 


\section{Total de estudos encontrados: 516}

Academic Search Complete ( $n=143)$; PsycARTICLES ( $n=33)$; PsycINFO $(n=303)$; Psychology and Behavioral Sciences Collection $(n=37)$.

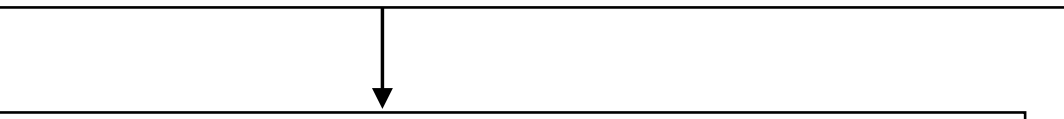

\section{Estudos excluídos: 454}

Duplicados ( $n=286)$; constrangimentos linguísticos $(n=2)$; não disponibiliza full text $(n=24)$; teses / dissertações $(n=66)$; comentários $(n=2)$; livros $(n=12)$; sem relação com as questões de investigação $(n=13)$; variáveis perdão e satisfação conjugal não contempladas simultaneamente $(n=49)$.

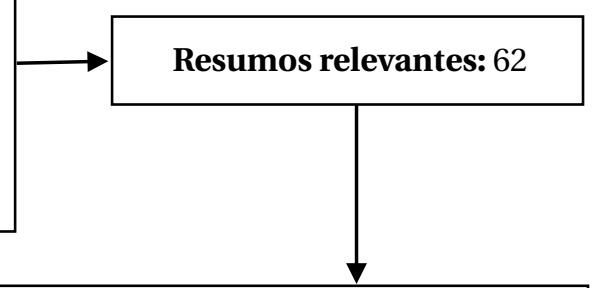

\section{Estudos excluídos: 37}

Tempo relação inferior a 2 anos $(n=15)$; indivíduos não envolvidos numa relação romântica $(n=1)$; variáveis perdão e satisfação conjugal não contempladas simultaneamente $(\mathrm{n}=8)$; amostra constituída apenas por um participante $(n=1)$; artigo de opinião $(n=2)$; revisão de literatura $(n=1)$; sem relação com as questões de investigação

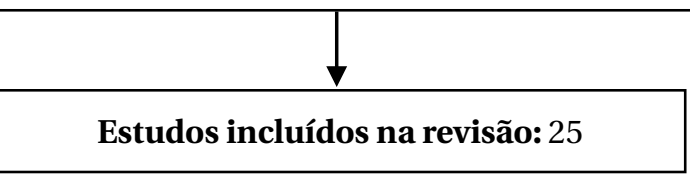

Figura 1

Diagrama de fluxo que descreve o processo de revisão sistemática

Em relação à conceptualização do perdão, verifica-se um destaque para uma abordagem teórica e definição do construto numa dimensão multidimensional, emergindo uma dimensão positiva (benevolência) e negativa (evitamento, vingança ou retaliação), sendo que apenas sete estudos não apresentaram uma definição conceitual (Kim, Johnson, \& Ripley, 2011; McNulty \& Russell, 2016; Merolla \& Shuang Yue, 2011; Myung-Sun, 2014; Olson, Marshall, Goddard, \& Schramm, 2015; Schumann, 2012; Sheldon, Gilchrist-Petty, \& Lessley, 2014). O estudo de Pelucchi, Paleari, Regalia e Fincham (2013) também conceptualiza o autoperdão (forgiveness of self ou benevolência e compaixão perante o self) e unforgiveness of self (ausência de benevolência e compaixão, autopercepção negativa). Por sua vez, dois estudos desdobram o construto em três níveis específicos, nomeadamente, o perdão enquanto traço de personalidade, como uma tendência específica num relacionamento (ou perdão diádico) e como uma resposta a uma ofensa específica (ou perdão episódico) (Allemand et al., 2007; Paleari, Regalia, \& Fincham, 2009).
No que concerne às relações entre perdão e satisfação conjugal (Quadro 1), embora a grande maioria dos estudos não possuísse como objetivo exclusivo analisar de que modo ambos estão relacionados, como reflexo da própria heterogeneidade dos objetivos dos estudos incluídos, verificou-se em três deles que o perdão está positivamente correlacionado com a satisfação conjugal (Allemand et al., 2007; Orathinkal \&Vansteenwegen, 2006; Pansera \& La Guardia, 2012) e em seis estudos que o perdão foi um preditor positivo da satisfação conjugal (Braithwaite et al., 2011; David \& Stafford, 2015; Gordon, Hughes, Tomcik, Dixon, \& Litzinger, 2009; Myung-Sun, 2014; Olson et al., 2015; Patrick, Beckenbach, Sells, \& Reardon, 2013). Ao considerar as diferentes dimensões do perdão, quatro estudos revelaram que a dimensão positiva (benevolência) e negativa do perdão (vingança, retaliação e evitamento) estão positiva e negativamente correlacionadas, respectivamente, com a satisfação conjugal (Bugay, 2014; David \& Stafford, 2015; Paleari et al., 2009; Stafford, David, \& McPherson, 2014). Já em relação a outros tipos de perdão, dois estudos verifi- 
caram que o autoperdão está também positivamente associado com a satisfação conjugal de ambos os parceiros (Pelucchi et al., 2013; Thompson et al., 2005).

No que se refere às variáveis mediadoras, verificou-se nos dois estudos de Braithwaite et al. (2011) que a ligação entre o perdão e a satisfação conjugal é mediada pelo aumento do esforço relacional e diminuição das táticas negativas de resolução de conflitos, por exemplo, agressão física. Já o estudo de Schumann (2012) revelou que as percepções acerca da sinceridade do pedido de desculpas mediaram a associação entre a satisfação conjugal e o perdão, enquanto o estudo de Fincham, Paleari e Regalia (2002) revelou uma cadeia causal, onde a satisfação conjugal foi um preditor positivo das atribuições benignas. Por sua vez, as atribuições de responsabilidade positivas estavam diretamente relacionadas com o perdão e, indiretamente, a mediação das reações afetivas negativas e da empatia, pelo que os cônjuges que fazem atribuições mais positivas são mais empáticos e experienciam menos afetos negativos.

Apenas o estudo de Fincham e Beach (2007) teve como objetivo exclusivo analisar possíveis relações causais entre o perdão e a satisfação conjugal, revelando correlações significativas positivas, tanto em nível transversal como longitudinalmente. Os mesmos autores verificaram um efeito causal bidirecional entre o perdão e a satisfação conjugal para as mulheres e um efeito causal unidirecional para os homens (da satisfação conjugal ao perdão). Já o estudo de Paleari et al. (2005) indicou, em nível concorrente, que cônjuges que apresentam altos níveis de empatia emocional e níveis inferiores de ruminação em relação à ofensa revelam níveis superiores de perdão, estando ainda mais satisfeitos com o relacionamento conjugal. Verificou-se também que a trajetória da ruminação e do não-perdão foi mais forte para as mulheres do que para os homens, enquanto a trajetória da empatia e benevolência foi mais forte para os homens do que para as mulheres. Os autores também demonstraram uma relação indireta recíproca entre o perdão e a satisfação conjugal ao longo do tempo, em que cônjuges que estavam mais satisfeitos com o casamento perdoam mais o parceiro seis meses depois por ruminarem menos sobre a ofensa.

Emergiram ainda outras questões relacionadas às diferenças de gênero. O estudo de Bugay (2014) revelou que, comparadas aos homens, as mulheres apresentaram scores significativamente mais baixos na dimensão negativa do perdão e scores significativamente superiores na dimensão positiva. Paleari et al. (2009) também demonstraram que a benevolência foi um preditor positivo mais forte da satisfação conjugal nas mulheres do que nos homens. Por fim, surgiram estudos em que os resultados não foram foco principal de análise. Contudo, uma breve descrição merece ser feita por sua possível pertinência. Seis deles revelaram que o perdão está associado a uma resolução de conflitos mais positiva (Braithwaite et al., 2011; Fincham, Beach, \& Davila, 2004; Fincham et al., 2007; Gordon et al., 2009; Paleari et al., 2009; Patrick et al., 2013); dois estudos revelaram que o uso de estratégias de comunicação diretas (perdoar de forma explícita) (Merolla \& Shuang Yue, 2011; Sheldon et al., 2014), não-verbais (abraços) e minimizadoras do perdão (minimização das consequências ofensa) (Sheldon et al., 2014) estavam positivamente associadas à satisfação conjugal. Por sua vez, um estilo de comunicação condicional (perdoar apenas com a imposição de condições) estava negativamente associado (Merolla \& Shuang Yue, 2011; Sheldon et al., 2014). Desta forma, o estudo de Gordon et al. (2009) revelou que o perdão está positivamente associado a uma aliança parental mais forte e a percepções mais positivas por parte dos filhos sobre o funcionamento conjugal dos pais.

\section{Discussão}

Iniciando pelas características metodológicas, verifica-se que todos os estudos incluídos nesta revisão foram publicados entre os anos de 2002 e 2016, acentuando a ideia de que a investigação do perdão no âmbito dos relacionamentos românticos é recente. Os estudos foram realizados em diferentes contextos culturais, com predominância da América do Norte, particularmente nos Estados Unidos. Parece inevitável refletir sobre os efeitos relacionados a padrões culturais que possam influenciar os resultados obtidos. Curiosamente, o estudo de Bugay (2014), realizado na Turquia, revelou que as mulheres apresentavam níveis superiores de perdão na relação, suportando o resultado com o facto de, na cultura turca, as mulheres serem encorajadas a manter a harmonia no casamento, enquanto os homens revelam maior poder social e familiar. Sendo assim, o maior perdão por parte das esposas poderia ser reflexo de um status social inferior. Deste modo, levanta-se a questão se um mesmo construto, particularmente o perdão, encerra em si diferentes significados, conforme o contexto social e cultural em que se insere. 
Face aos objetivos dos estudos incluídos, constata-se a heterogeneidade, com destaque para a evolução interdisciplinar que a investigação no âmbito do perdão está a tomar (Fehr et al., 2010), parecendo que essa diversidade se traduz num interesse pela compreensão das mais diversas variáveis e suas possíveis influências ao nível do perdão.

Relativamente às definições conceptuais do perdão utilizadas nos estudos, constatou-se que a grande maioria apresentou uma definição ou reflexão delas, existindo clara preferência por uma abordagem multidimensional e motivacional do construto. Ou seja, destaque não só para a renúncia de um estado motivacional negativo (dimensão negativa caracterizada pelo ressentimento, evitamento ou distanciamento e vingança), mas também para a presença de um estado motivacional positivo (dimensão positiva caracterizada pela bene- volência). O mesmo se verificou no caso de outro tipo de perdão, nomeadamente, o autoperdão (Pelucchi et al., 2013), que vai ao encontro da literatura, que parece estar a se encaminhar, consensualmente, para uma definição multidimensional do perdão (Enright \& The Human Development Study Group, 1991). Esse maior consenso conceitual também pode ser relevante na adaptação e desenvolvimento de medidas de avaliação do perdão, de modo que sua operacionalização não se traduz na literatura num conjunto de instrumentos de avaliação contraditórios. Dois estudos foram além (Allemand et al., 2007; Paleari et al., 2009), diferenciando o perdão em três níveis específicos. Parece, assim, que o perdão não é um fenômeno igualitário, podendo variar de indivíduo para indivíduo (perdão enquanto traço de personalidade), conforme o tipo de relação (perdão diádico) e conforme a ofensa (perdão episódico).

\section{Quadro 1}

Principais resultados dos estudos analisados.

\section{Id. Principais resultados}

1 Operfeccionismo socialmente prescrito e o neuroticismo emergiram como os únicos preditores inversamente significativos do perdão do outro. O perfeccionismo auto-orientado e o neuroticismo emergiram como preditores significativos da satisfação conjugal.

2 O próprio perdão e o perdão do cônjuge positivamente associados com a satisfação conjugal individual e do parceiro, enquanto a falta de perdão entre os cônjuges estão negativamente associados com a satisfação conjugal.

3 Demonstração de benevolência, justiça, empatia, confiança e perdão relacionadas com a satisfação conjugal. A satisfação conjugal se revelou uma variável subsequente ao perdão.

4 Perdão e pensamento hostil demonstraram associações equivalentes, embora inversas, com a duração da relação e o perdão, explicando a variância única na satisfação do relacionamento, mesmo quando controlada a confiança.

5 Satisfação com o relacionamento negativamente associada à gravidade da ofensa. Pedido de desculpas fortemente associado com o perdão apenas nos casais altamente satisfeitos com seus relacionamentos. Percepções sobre a sinceridade do pedido de desculpas mediaram a associação entre a satisfação com o relacionamento e o perdão. A satisfação com o relacionamento não foi significativamente associada ao perdão quando o pedido de desculpas estava ausente. No entanto, quando estava presente, a satisfação com o relacionamento foi positivamente associada ao perdão.

6 A afabilidade moderou os efeitos da percepção do perdão do parceiro em transgressões contra esse parceiro. Perdão do parceiro negativamente associado à ofensa subsequente entre pessoas mais afáveis, mas positivamente associado à ofensa subsequente entre as pessoas menos afáveis.

7 Estudo 1: Foram identificadas duas dimensões do perdão, uma positiva (benevolência) e outra negativa (retaliação), que estão associadas à resolução de conflito nos casais. Uma resolução ineficaz dos conflitos se relaciona significativamente com a própria satisfação conjugal e a satisfação conjugal do parceiro.

Estudo 2: Foram identificadas três dimensões do perdão (retaliação, evitamento e benevolência).

8 Satisfação conjugal e perdão relacionados entre si, concorrente e longitudinalmente. Para as mulheres, emergiram trajetórias significativas do perdão à satisfação conjugal e vice-versa (efeito bidirecional). Já em relação aos homens, a direção do efeito era da satisfação conjugal ao perdão (efeito unidirecional). Efeito indireto significativo para as mulheres, mas não para os homens, indicando que a satisfação conjugal concorrente mediou o efeito da satisfação anterior do casamento no perdão posterior para as esposas, mas não para os maridos. 
9 Estudo 1: Perdão não diretamente relacionado à satisfação conjugal, mas, sim, com o aumento do esforço relacional e à diminuição dos comportamentos interpessoais negativos, que, por sua vez, estavam relacionados com a satisfação conjugal. Existiu um efeito indireto significativo, de tal modo que o esforço relacional e as táticas negativas mediaram a relação entre perdão e satisfação conjugal.

Estudo 2: Associações entre o perdão, os mediadores propostos e a satisfação com o relacionamento se mantêm longitudinalmente. A tendência para perdoar está relacionada com a satisfação conjugal posterior.

10 Satisfação conjugal significativamente relacionada às atribuições de responsabilidade, direta e indiretamente por meio das atribuições causais. Atribuições de responsabilidade relacionadas com o perdão tanto direta como indiretamente por meio das reações afetivas negativas e da empatia emocional. Satisfação conjugal não diretamente associada ao perdão, às reações afetivas ou à empatia emocional.

11 Estudo 1: Perdão positivamente associado com mudanças nos níveis do compromisso no relacionamento e negativamente associado com a severidade da transgressão.

Estudo 2: Perdão positivamente associado com mudanças nos níveis do compromisso no relacionamento e negativamente associado com os autorrelatos da severidade da transgressão percebida.

12 Os estilos de comunicação do perdão dos parceiros (isto é, direto, indireto e condicional) são influenciados por variáveis da ofensa e do ofensor.

O perdão direto diminuiu o dano relacional, que, por sua vez, aumentou a satisfação conjugal. O perdão direto também está diretamente e positivamente associado com a satisfação conjugal. O perdão condicional previu positivamente os danos relacionais - o perdão condicional prejudicou as relações (isto é, aumentou o dano relacional). O perdão indireto não está relacionado com os danos relacionais.

13 Relação longitudinal entre perdão e resolução de conflitos; especificamente, esposas que demonstravam uma menor benevolência face às transgressões dos companheiros, relataram níveis mais elevados de uma resolução ineficaz de conflitos doze meses mais tarde. Já para os parceiros do sexo masculino, o único preditor significativo dos relatos das esposas acerca de uma resolução posterior de conflitos ineficaz foi o nível inicial de resolução de conflitos.

14 A ruminação e a empatia emocional estão concorrentemente relacionadas com o perdão, que, por sua vez, parece afetar a satisfação conjugal.

As trajetórias que ligam as variáveis sociocognitivas ao perdão e à satisfação conjugal sugerem a existência de algumas diferenças de gênero. Pensamentos e sentimentos ruminativos parecem promover mais a falta de perdão entre as esposas do que entre os maridos Inversamente, a trajetória que relaciona a empatia com a benevolência pareceu mais forte para os maridos do que para as esposas. Uma relação recíproca e indireta foi obtida entre perdão e satisfação conjugal ao longo do tempo.

15 Estudo 1: Escala final revelou consistência interna adequada, emergindo duas dimensões distintas correlacionadas, positiva (benevolência) e negativa (ressentimento-evitação). Cônjuges que eram mais benevolentes e menos ressentidos ou evitantes em resposta à ofensa tinham relacionamentos conjugais mais satisfatórios, próximos e de apoio do que os cônjuges menos benevolentes.

Estudo 2: A única diferença significativa da benevolência foi que ela serviu como um preditor mais forte da própria satisfação conjugal para as esposas do que para os maridos. Para ambos os cônjuges, a benevolência previu positivamente o autorrelato da satisfação conjugal

Estudo 3: Menor ressentimento e evitamento associados à percepção de um casamento mais próximo e satisfatório, maior eficácia na resolução de conflitos e confiança no parceiro.

16 A correlação entre os scores da Marital Dispositional Forgiveness Scale (MDFS) e da Relationship Satisfaction Scale (RAS) foi significativa, sugerindo uma relação positiva entre o perdão e a satisfação conjugal. Especificamente, a dimensão positiva esteve positivamente correlacionada com a satisfação conjugal e a dimensão esteve correlacionada negativamente com a satisfação conjugal entre ambos os cônjuges. Mulheres apresentaram scores significativamente mais baixos na dimensão negativa do perdão em relação aos homens e scores significativamente superiores na dimensão positiva do perdão, comparativamente com os homens.

17 O perdão foi um preditor positivo da satisfação conjugal. 
Id. Principais resultados

18 Perdão positivamente associado com a satisfação conjugal e negativamente associado com a insatisfação conjugal. Não-perdão negativamente associado com a satisfação conjugal e positivamente associado com a insatisfação conjugal.

19 Identificadas duas dimensões distintas do autoperdão: dimensão positiva, que reflete a benevolência e compaixão para com o self (forgiveness of self), e dimensão negativa, que caracteriza a falta de benevolência e compaixão para com o self, autorressentimento e auto-visão negativa (unforgiveness of self). Quanto mais o ofensor é benevolente para consigo mesmo, mais satisfeito está com o relacionamento conjugal. Quanto mais o ofensor sente emoções negativas contra o self, menos satisfeito está com o relacionamento. Pensamentos e sentimentos positivos por parte do ofensor em relação ao self estão associados a uma maior satisfação no relacionamento com os parceiros ofendidos

20 Homogamia religiosa, oração pelo bem-estar do cônjuge e perdão do cônjuge predizem significativamente níveis mais elevados de satisfação conjugal.

21 Não se verificou diferenças estatisticamente significativas ao nível do perdão entre os casais que se encontravam no primeiro casamento e recasados. Existe uma diferença significativa ao nível da satisfação conjugal entre os casais que se encontravam no primeiro casamento e os recasados, sendo os indivíduos recasados que indicam maiores níveis de satisfação conjugal. Existe uma correlação significativa positiva entre o perdão e satisfação conjugal.

22 Os participantes perdoavam menos quanto maior fosse a severidade da ofensa, quanto mais percebiam o parceiro como responsável e as ações do parceiro como intencionais. Quanto menos os participantes estavam satisfeitos e comprometidos com o relacionamento, menos perdoavam os parceiros. O modelo de mediação é moderado pela gravidade da ofensa e pela satisfação com o relacionamento.

23 Associação significativa positiva entre perdão-traço e a satisfação conjugal. Associação positiva significativa entre perdão episódico e a satisfação conjugal.

24 Perdão prediz a satisfação conjugal. Um perdão mais negativo foi associado com uma menor satisfação conjugal para ambos os cônjuges. Um maior perdão positivo previu os próprios relatos de uma aliança parental mais forte, enquanto um maior perdão negativo está associado a uma aliança parental mais fraca.

25 Parceiros com maior tendência para perdoar estão mais satisfeitos nos relacionamentos. Não existiu uma relação significativa entre a tendência para perdoar e a satisfação conjugal em parceiros envolvidos em relações de namoro. Parceiros que utilizaram estratégias não-verbais e minimizadoras estavam mais satisfeitos com a relação, enquanto aqueles que usaram uma abordagem condicional estavam menos satisfeitos. Nos parceiros em relações de namoro, aqueles que usavam estratégias explícitas estavam mais satisfeitos, enquanto aqueles que usavam abordagens condicionais estavam menos satisfeitos.

Quanto às relações entre os construtos perdão e satisfação conjugal, foi confirmada uma associação positiva, constatando-se correlações positivas em três estudos (Allemand et al., 2007; Orathinkal \& Vansteenwegen, 2006; Pansera \& La Guardia, 2012) e o valor preditivo positivo do perdão em seis estudos, sendo que quanto mais os parceiros românticos perdoam, maior será a satisfação conjugal percepcionada (Braithwaite et al., 2011; David \& Stafford, 2015; Gordon et al., 2009; Myung-Sun, 2014; Olson et al., 2015; Patrick et al., 2013). Novamente, realça-se a consideração das duas diferentes dimensões do perdão em quatro estudos, apresentando correlações distintas com a satisfação conjugal (Bugay, 2014; David \& Stafford, 2015; Paleari et al., 2009; Stafford et al., 2014), o que denota, mais uma vez, a importância de se realizar essa distin- ção e considerar a natureza multidimensional do perdão. Tal como a investigação tem sugerido, denota-se, assim, que o perdão está associado a resultados positivos em relacionamentos próximos (Burr et al., 2012), resultado que parece se traduzir na promoção da satisfação conjugal do casal. Na mesma linha, o autoperdão também apresentou uma relação positiva com a satisfação conjugal (Pelucchi et al., 2013; Thompson et al., 2005), sendo que os benefícios parecem repercutir não só no ofensor, como também no ofendido, o que apoia a perspetiva de o perdão possuir elementos tanto intra como interpessoais (McCullough et al., 2003). Revela-se, assim, importante não existir uma focalização apenas na perspetiva da vítima, considerando-se também a perspetiva do ofensor e o todo que constitui a relação. 
Embora sejam evidentes as relações positivas encontradas entre os dois construtos, permanece na literatura a dúvida sobre os mecanismos ou processos subjacentes à relação entre ambos. Alguns estudos parecem ter fornecido algumas respostas, como é o caso do trabalho de Braithwaite et al. (2011), no qual a ligação entre o perdão e a satisfação conjugal é explicada pelo fato de parceiros que revelam uma maior tendência para perdoar serem mais propensos a se esforçarem na relação e a inibir estratégias de resolução de conflito negativas, o que, por sua vez, promove níveis superiores de satisfação conjugal. Parece, assim, que os resultados positivos do perdão podem também advir de uma resolução mais eficaz das situações de conflito que emergem no casal, possibilitando a interrupção dos ciclos de reciprocidade negativa. Noutra linha, o estudo de Schumann (2012) revelou que casais mais satisfeitos com o relacionamento conjugal perdoavam mais face a um pedido de desculpas por entenderem esse pedido como uma expressão sincera de arrependimento. É importante relembrar que os casais satisfeitos se pautam por uma dinâmica em que as cognições, emoções e comportamentos positivos prevalecem sobre os negativos (Gottman \& Silver, 2001). Sendo assim, as tentativas por parte dos parceiros ofensores para atenuar as consequências das suas ofensas (como a presença de um pedido de desculpas) podem ser encaradas como gestos genuínos de arrependimento e boa vontade. Na mesma linha, o estudo de Fincham et al. (2002) revelou que parceiros mais satisfeitos nos relacionamentos tendem a perdoar mais porque realizam atribuições mais positivas face à situação ofensiva, o que, por sua vez, tem um efeito positivo direto no perdão, mas também indireto, pois parceiros que realizam atribuições mais positivas são mais empáticos e experienciam menos afeto negativo, o que, por sua vez, promove níveis superiores de perdão. Assim, os resultados dos três estudos supracitados parecem indicar que não só o perdão pode promover e aumentar os níveis de satisfação conjugal no casal, como a satisfação conjugal, nomeadamente, pode percepcionar níveis superiores nessa dimensão e promover um maior perdão na díade conjugal. No entanto, parece que a relação entre perdão e satisfação conjugal se revela mais indireta do que direta, como resultado da influência de fatores de natureza sociocognitiva e relacionados à natureza da ofensa, o que corrobora a natureza distal dos determinantes do relacionamento.
Apenas o estudo de Fincham e Beach (2007) teve como objetivo exclusivo analisar as relações causais entre o perdão e a satisfação conjugal, revelando correlações positivas entre os construtos, tanto transversal como longitudinal. Contudo, não foram analisados que possíveis mecanismos possam estar subjacentes a essa associação. Já o estudo longitudinal de Paleari et al. (2005) demonstrou que quanto mais os parceiros empatizam com o ofensor e menos ruminam sobre a ofensa, revelam níveis superiores de perdão, estando ainda mais satisfeitos com o relacionamento conjugal. Da mesma forma, demonstraram uma relação recíproca entre o perdão e a satisfação conjugal ao longo do tempo, em que cônjuges que estavam mais satisfeitos com o casamento perdoavam mais o parceiro por ruminarem menos sobre a ofensa. A relação entre perdão e satisfação conjugal neste estudo foi também bidirecional, revelando-se mais indireta do que direta, como resultado da influência de fatores de natureza sociocognitiva, seja transversal ou longitudinal.

Diferenças de gênero podem ainda ser constatadas. No estudo de Paleari et al. (2009), a dimensão da benevolência foi um preditor positivo mais forte da satisfação conjugal nas mulheres do que nos homens. Já o estudo de Fincham e Beach (2007) revelou um efeito causal bidirecional entre o perdão e a satisfação conjugal para as mulheres, e um efeito causal unidirecional para os homens, ao passo que, no estudo de Paleari et al. (2005), se constatou que a trajetória da ruminação e do não-perdão (dimensão negativa) foi mais forte para as mulheres, enquanto a trajetória da empatia e benevolência (dimensão positiva) foi mais forte para os homens. As diferenças nesses estudos parecem, de modo geral, irem ao encontro da literatura, que postula que as mulheres são mais inclinadas a perdoar (Finkel et al., 2002; Miller et al., 2008; Orathinkal \& Vansteenwegen, 2008) e mais orientadas para os relacionamentos (Hook et al., 2003), sendo possível se beneficiarem mais das consequências positivas do perdão (traduzindo-se na sua satisfação conjugal), mas, também, levar o sexo feminino a se responsabilizar mais pela resolução das transgressões que surgem no relacionamento e a uma procura mais ativa de possíveis estratégias que permitam essa resolução e influenciem positivamente o relacionamento, podendo o perdão ser uma delas, compreendendo-se assim no estudo de Fincham e Beach (2007) a maior interação recíproca 
entre o perdão e a satisfação conjugal nas mulheres comparativamente com os homens, em que os níveis de satisfação conjugal que predizem o perdão. Por outro lado, esse maior sentido de responsabilidade por parte das mulheres na procura de explicações e soluções para as situações conflituosas poderá se tornar excessivo, recaindo numa vivência de afetos e cognições negativas e intrusivas relacionadas com a ofensa, o que é característico da ruminação. De fato, outras vertentes da investigação afirmam que, em relação aos homens, as mulheres apresentam maiores níveis de raiva nos relacionamentos (Gonzales, Haugen, \& Manning, 1994), o que poderia suportar a trajetória da ruminação nas mulheres no estudo de Paleari et al. (2005).

No entanto, há que ter em conta que as representações acerca dos papéis de gênero estão constantemente em mudança. Cada amostra possui características particulares suscetíveis de influenciar os resultados obtidos, além de ser fundamental considerar a influência de outros fatores, nomeadamente, culturais e sociais, que moldam os papéis de gênero.

Seis estudos ainda confirmaram a importância e efeito positivo do perdão no âmbito do conflito (Braithwaite et al., 2011; Fincham et al., 2004; Fincham et al., 2007; Gordon et al., 2009; Paleari et al., 2009; Patrick et al., 2013), tal como a importância do diálogo em dois estudos (Merolla \& Shuang Yue, 2011; Sheldon et al., 2014) como forma de levar a cabo estratégias que permitam a concessão do perdão, que, por sua vez, terão implicações na satisfação conjugal do casal. De fato, a literatura postula que fatores comportamentais presentes nas interações conjugais, tal como a comunicação, influenciam a satisfação (Halford \& Moore, 2002). Por fim, o perdão não está só positivamente associado à satisfação da díade conjugal, mas também é capaz de promover uma aliança parental mais forte e percepções mais positivas dos filhos sobre o funcionamento conjugal dos pais, o que foi evidente no estudo de Gordon et al. (2009). Parece, assim, que a existência de ofensas por resolver na díade não interfere negativamente apenas na esfera do casal, transpondo-se para outros subsistemas familiares, nomeadamente, o parental, expondo os filhos aos conflitos dos progenitores. Certamente, isso terá implicações negativas para o bem-estar emocional dos filhos, uma vez que os pais representam modelos importantes de funcionamento interpessoal.
Os estudos analisados nesta revisão sistemática possuem várias limitações metodológicas comuns, como a limitação associada à generalização dos resultados (amostras obtidas por conveniência), o viés típico associado à utilização de medidas de autorrelato, ou o próprio método de recolha de dados, incidindo apenas na perspetiva do parceiro ofendido. Contudo, é fundamental ultrapassar essa perspetiva unilateral e compreender a própria dinâmica da relação, já que o perdão parece operar de modo distinto para ambos os cônjuges (Fincham et al., 2005).

Destaca-se ainda o fato de a maioria dos estudos apresentar um design metodológico transversal. Dos 25 artigos analisados, quatro apresentam design metodológico longitudinal (Fincham \& Beach, 2007; Fincham et al., 2007; Paleari et al., 2005; Ysseldyk \& Wohl, 2012) e um transversal e longitudinal (Braithwaite et al., 2011). Não negligenciando o valor dos resultados obtidos dos estudos transversais, a escassez de estudos longitudinais levanta preocupações significativas, pois permitiriam uma visão mais precisa das possíveis relações entre as variáveis ao longo do tempo, uma vez que a pesquisa transversal não elimina a ambiguidade direcional das relações causais das variáveis (McNulty, 2008), tornando-se, assim, difícil retirar conclusões rigorosas e, ainda, dos possíveis mecanismos que podem estar subjacentes. Clarificando, embora não seja o design metodológico que forneça as informações sobre as relações de causa-efeito, uma vez que isso depende dos procedimentos estatísticos utilizados, o estabelecimento de uma precedência temporal se revela fundamental para inferir causalidade e compreender com maior certeza as relações e direções das variáveis, uma vez que a literatura continua com várias questões por resolver sobre as possíveis relações entre os construtos perdão e satisfação conjugal (Allemand et al., 2007; Braithwaite et al., 2011; Fincham \& Beach, 2007).

\section{Considerações finais}

Esta revisão teve como principal objetivo a síntese da evidência científica de estudos que fazem referência simultânea aos construtos perdão e satisfação conjugal em relacionamentos românticos adultos, contribuindo, assim, para uma melhor integração empírica da literatura existente. Os estudos incluídos nesta revisão remetem a publicações recentes, existindo uma heterogeneidade dos obje- 
tivos, denotando, assim, um interesse crescente pela área do perdão na conjugalidade. As definições conceptuais apresentadas pelos estudos revelaram claramente que o perdão, no âmbito de relacionamentos românticos, só pode ser verdadeiramente compreendido segundo uma abordagem multidimensional, comportando uma dimensão positiva e negativa do construto. Torna-se, assim, relevante continuar a caminhar no sentido de uma maior higiene conceitual, que é fundamental na própria operacionalização do perdão.

Os principais resultados revelaram, de um modo geral, que o perdão pode funcionar tanto como um precursor (um maior perdão é capaz de promover níveis superiores de satisfação conjugal), bem como uma consequência de relacionamentos conjugais satisfeitos (níveis superiores são capazes de fomentar um maior perdão na díade conjugal). Ademais, essa relação pode ser bidirecional, em que o perdão e a satisfação conjugal são suscetíveis de se influenciarem mutuamente. Contudo, continua por explorar e compreender que possíveis mecanismos podem estar subjacentes a essa relação, pois poucos estudos avançaram com alguma compreensão nesse campo, constatando-se que a relação entre perdão e satisfação conjugal é mais indireta do que direta, sendo resultado da influência de diversos fatores. Persiste, do mesmo modo, a necessidade de ultrapassar diversas limitações metodológicas que dificultam essa compreensão para a retirada de conclusões seguras, como é o caso do design metodológico dos estudos incluídos nessa revisão, revelando-se predominantemente transversal, confirmando a negligência da dimensão temporal característica da investigação no âmbito do perdão (McCullough et al., 2003). Apelamos, assim, que investigações futuras apostem nessa temporalidade.

Esperamos que os resultados desta revisão sistemática possam ter implicações para a prática psicológica, particularmente no âmbito da clínica com casais e famílias. Sendo o perdão um fenômeno intra e interpessoal, é fundamental compreender as expectativas que cada membro traz para a relação, assim como explorar e reconstruir significados associados ao perdão, já que a vítima e o ofensor possuem perspectivas distintas face à situação ofensiva. De fato, comunicar "perdoei-te" pode significar o início do processo de perdão para o ofendido, mas pode ser compreendido pelo ofensor como o cessar absoluto do acontecimento ofensivo.
Trabalhar com o casal essa questão é crucial, uma vez que a vivência da conjugalidade é naturalmente pautada por desafios, que podem ser causadores de conflito. Como tal, o perdão poderá ser utilizado como estratégia construtiva para a resolução de conflitos, psicoeducando o casal nesse sentido. De facto, as transgressões possuem motivos diversos, desde preferências incompatíveis, conflitos familiares ou, até, situações de infidelidade. Como tal, é fundamental que o próprio psicólogo possua um conhecimento aprofundado desse construto, sendo capaz de identificar em que etapa do processo de perdão o casal se encontra, se ainda persiste a necessidade de experienciar a dor emocional da ofensa ou se estão reunidas as condições para avançar para um novo conjunto de crenças sobre o relacionamento. É relevante reconhecer também quando o perdão se pode revelar uma limitação enquanto estratégia de intervenção terapêutica, respeitando-se assim as idiossincrasias de cada relação.

Este estudo não está isento de limitações. Embora tenha sido delineada uma estratégia de investigação rigorosa que permitisse uma pesquisa abrangente, subsiste um viés de publicação difícil de superar, pois nem todos os estudos estavam disponíveis, existindo assim a possibilidade de pesquisas relevantes não terem sido incluídas, o que certamente enriqueceria esta revisão. Conquanto, ainda que se tenha estabelecido como critério de inclusão indivíduos em relações românicas há pelo menos dois anos e em coabitação, é prudente reconhecer que tal critério não foi cumprido de forma rigorosa, pois alguns estudos não forneceram informações que permitissem aferir se os participantes estavam ou não em coabitação. Ademais, nossa decisão se baseou no valor médio do tempo de relacionamento (sempre que tal informação fosse fornecida pelos próprios artigos), com o intuito de evitar a perda de um grande número de artigos na amostra. Como tal, é possível existir um enviesamento dos resultados, sendo necessário prudência em sua generalização.

Finalizando, acreditamos que este estudo possa fornecer insights teóricos e metodológicos sobre a investigação do perdão na conjugalidade. O perdão é um construto essencial para promover uma relação saudável e satisfatória, independentemente da sua natureza. Como tal, apelamos a um continuado investimento da investigação, permitindo, cada vez mais, uma rigorosa integração empírica e progresso científico. 


\section{Referências}

Allemand, M., Amberg, I., Zimprich, D., \& Fincham, F. D. (2007). The role of trait forgiveness and relationship satisfaction in episodic forgiveness. Journal OfSocial and Clinical Psychology, 26(2), 199217. https://doi.org/10.1521/ jscp.2007.26.2.199

Barnett, J. K., \& Youngberg, C. (2004). Forgiveness as a ritual in Couples Therapy. The Family Journal, 12(1), 1420. https:/ / doi.org/ 10.1177/106648070325861

Braithwaite, S. R., Selby, E. A., \& Fincham, F. D. (2011). Forgiveness and relationship satisfaction: Mediating mechanisms. Journal of Family Psychology, 25(4), 551559. https://doi.org/10.1037/a0024526

Bugay, A. (2014). Measuring the differences in pairs' marital forgiveness scores: Construct validity and links with relationship satisfaction. Psychological Reports, 114(2), 479490. https://doi.org/10.2466/21.02.PR0.114k18w5

Burr, W. R., Marks, L. D., \& Day, R. D. (2012). Sacred matters: Religion and spirituality in families. Routledge.

David, P., \& Stafford, L. (2015). A relational approach to religion and spirituality in marriage: The role of couples' religious communication in marital satisfaction. Journal of Family Issues, 36(2), 232249. https:// doi.org/10.1177/0192513X13485922

Enright, R. D., \& The Human Development Study Group. (1991). The moral development of Forgiveness. In W. M. Kurtines, \& J. L. Gewirtz (Eds.), Handbook of moral behavior and development: Volume 1: Theory (pp. 123152). Lawrence Erlbaum.

Fehr, R., Gelfand, M., J, \& Nag, M., (2010). The road to forgiveness: A meta-analytic synthesis of its situational and dispositional correlates. Psychological Bulletin, 136(5), 894914. https://psycnet.apa.org/doi/10.1037/a0019993

Fenell, D. L. (1993). Characteristics of longer-term first marriages. Journal of Mental Health and Counseling, 15(4), 446460. https://psycnet.apa.org/record/1994-09260-001

Fincham, F. D., \& Beach, S. R. H. (2002). Forgiveness in marriage: Implications for psychological aggression and constructive communication. Personal Relationships, 9(3), 239251. https://doi.org/10.1111/1475-6811.00016

Fincham, F. D., \& Beach, S. R. H. (2007). Forgiveness and marital quality: Precursor or consequence in well-established relationships? The Journal of Positive Psychology, 2(4), 260268. https:// doi.org/10.1080/17439760701552360

Fincham, F. D., Beach, S. H., \& Davila, J. (2004). Forgiveness and conflict resolution in marriage. Journal of Family Psychology, 18(1), 7281. https://doi.org/10.1037/0893-3200.18.1.72

Fincham, F. D., Beach, S. H., \& Davila, J. (2007). Longitudinal relations between forgiveness and conflict resolution in marriage. Journal of Family Psychology, 21(3), 542545. https://doi.org/10.1037/0893-3200.21.3.542

Fincham, F. D., Hall, J. H., \& Beach, S. R. H. (2005). Til lack of forgiveness doth us part: Forgiveness in marriage. In E. L. Worthington (Ed.), Handbook of forgiveness (pp. 207226). Routledge.

Fincham, F. D., Hall, J., \& Beach, S. R. H. (2006). Forgiveness in marriage: Current status and future directions. Family Relations: An Interdisciplinary Journal of Applied Family Studies, 55(4), 415427. https://doi. org/10.1111/j.1741-3729.2005.callf.x-i1

Fincham, F. D., Paleari, F. G., \& Regalia, C. (2002). Forgiveness in marriage: The role of relationship quality, attributions, and empathy. Personal Relationships, 9(1), 2737. https://doi.org/10.1111/1475-6811.00002

Finkel, E. J., Rusbult, C. E., Kumashiro, M., \& Hannon, P. A. (2002). Dealing with betrayal in close relationships: Does commitment promote forgiveness? Journal of Personality \& Social Psychology, 82(6), 956974. https:// doi.org/10.1037//0022-3514.82.6.956

Gonzales, M. H., Haugen, J. A., \& Manning, D. J. (1994).Victims as "narrative critics": Factors influencing rejoinders and evaluative responses to offenders' accounts. Personality and Social Psychology Bulletin, 20(6), 691704. https://psycnet.apa.org/doi/10.1177/0146167294206007

Gordon, K. C., \& Baucom, D. H. (1998). Understanding betrayals in marriage: A synthesized model of forgiveness. Family Process, 37, 425449. https://doi.org/10.1111/j.1545-5300.1998.00425.x

Gordon, K. C., Hughes, F. M., Tomcik, N. D., Dixon, L. J., \& Litzinger, S. C. (2009). Widening spheres of impact: The role of forgiveness in marital and family functioning. Journal of Family Psychology, 23(1), 113. https://doi. $\operatorname{org} / 10.1037 / \mathrm{a} 0014354$ 
Gottman, J. M., \& Silver, N. (2001). Os sete princípios do casamento. Pergaminho.

Guzmán-González, M., Alfaro, I., \& Armenta, C. (2013). Pérdon y satisfacción marital: Una mirada desde lo sistémico. Salud \& Sociedad, 4(3), 284294.

Halford, W. K., \& Moore, E. N. (2002). Relationship education and the prevention of couple relationship problems. In A. S. Gurman, \& N. S. Jacobson (Eds.), Clinical handbook of couple therapy (3a ed., pp. 400419). Guilford.

Hernandez, J. A. E., \& Oliveira, I. M. B. (2003). Os componentes do amor e a satisfação. Psicologia: Ciência e Profissão, 21(3), 5869.

Higgins, J., \& Green, S (Eds.). (2011). Cochrane handbook for systematic reviews of interventions (version 5.1.0) [atualizado em março de 2011]. The Cochrane Collaboration. https:/ /handbook-5-1.cochrane.org/

Hook, M., Gerstein, L., Detterich, L., \& Gridley, B. (2003). How close are we? Measuringintimacy and examininggender differences. Journal of Counseling and Development, 81(4), 462472. https://doi.org/10.1002/j.1556-6678.2003. tb00273.x

Karremans, J. C., Van Lange, P. A. M., Ouwerkerk, J. W., \& Kluwer, E. S. (2003). When forgiving enhances psychological well-being: The role of interpersonal commitment. Journal of Personality and Social Psychology, 84(5), 10111026. https://psycnet.apa.org/doi/10.1037/0022-3514.84.5.1011

Kim, L. M., Johnson, J. L., \& Ripley, J. (2011). A "perfect" storm: Perfectionism, forgiveness, and marital satisfaction. Individual Differences Research, 9(4), 199209.

Leary, M. R., Springer, C., Negel, L., Ansell, E., \& Evans, K. (1998). The causes, phenomenology, and consequences of hurt feelings. Journal of Personality and Social Psychology, 74(5), 12251237. https://psycnet.apa.org/ doi/10.1037/0022-3514.74.5.1225

McCullough, M. E. (1997). Marital forgiveness: Theoretical foundations and an approach to prevention. Marriage and Family: A Christian Journal, 1(1), 77-93.

McCullough, M. E., Fincham, F. D., \& Tsang, J. (2003). Forgiveness, forbearance, and time: The temporal unfolding of transgression-related interpersonal motivations. Journal of Personality and Social Psychology, 84(3), 540557. https://doi.org/10.1037//0022-3514.84.3.540

McCullough, M., Rachal, K., Sandage, S., Worthington Jr, E. L., Brown, S., \& Hight, T. (1998). Interpersonal forgiving in close relationships: II. Theoretical elaboration and measurement. Journal of Personality and Social Psychology, 75(6), 15861603. https://psycnet.apa.org/doi/10.1037/0022-3514.75.6.1586

McNulty, J. K. (2008). Forgiveness in marriage: Putting the benefits into context. Journal of Family Psychology, 22(1), 171-175. https://doi.org/10.1037/0893-3200.22.1.171

McNulty, J. K., \& Russell, V. M. (2016). Forgive and forget, or forgive and regret? Whether forgiveness leads to less or more offending depends on offender agreeableness. Personality and Social Psychology Bulletin, 42(5), 616631. https://doi.org/10.1177/0146167216637841

Mead, M. (1970). “Marriage in two steps” The family. In H. A. Otto (Ed.), Search of a future (pp. 7584). AppletonCentury-Crofts.

Merolla, A. J., \& Shuang Yue, Z. (2011). In the wake of transgressions: Examining forgiveness communication in personal relationships. Personal Relationships, 18(1), 7995. https://doi.org/10.1111/j.1475-6811.2010.01323.x

Miller, A. J., Worthington Jr., E. L., \& McDaniel, M. A. (2008). Gender and forgiveness: A meta-analytic review and research agenda. Journal of Social and Clinical Psychology, 27(8), 843876. https://doi.org/10.1521/ jscp.2008.27.8.843

Mosmann, C., Wagner, A., \& Féres-Carneiro, T. (2006). Qualidade conjugal: Mapeando conceitos. Paidéia: Cadernos de Psicologia e Educação, 16(35), 315325. https://doi.org/10.1590/S0103-863X2006000300003

Murray, J. R. (2002). Forgiveness as a therapeutic option. The Family Journal: Counseling and Therapy for Couples and Families, 10(3), 315321. https://doi.org/10.1177\%2F10680702010003008

Myung-Sun, C. (2014). Pathways between attachment and marital satisfaction: The mediating roles of rumination, empathy, and forgiveness. Personality \& Individual Differences, 70, 246251. https://doi.org/10.1016/j. paid.2014.06.032 
Noller, P., \& Feeney, J. A. (1998). Communication in early marriage: Response to conflict, nonverbal accuracy, and conversational patterns. In T. N. Bradbury (Ed.), The developmental course of marital dysfunction (pp. 1143). Cambridge University Press.

Norgren, M., Souza, R. M., Kaslow, F., Hammerschmidt, H., \& Sharlin, S. A. (2004). Satisfação conjugal em casamentos de longa duração: Uma construção possível. Estudos de Psicologia, 9(3), 575584. https://doi.org/10.1590/S1413294X2004000300020

Olson, D. H. (2000). Circumplex model of marital and family systems. Journal of Family Therapy, 22(2), 144167. https:// doi.org/10.1111/1467-6427.00144

Olson, J. R., Marshall, J. P., Goddard, H.W., \& Schramm, D. G. (2015). Shared religious beliefs, prayer, and forgiveness as predictors of marital satisfaction. Family Relations, 64(4), 519533. https://doi.org/10.1111/fare.12129

Orathinkal, J., \&Vansteenwegen, A. (2006). The effect of forgiveness on marital satisfaction in relation to marital stability. Contemporary Family Therapy: An International Journal, 28(2), 251260. https://doi.org/10.1007/s10591-006-9006-y

Paleari, F. G., Regalia, C., \& Fincham, F.D. (2005). Marital quality, forgiveness, empathy, and rumination:Alongitudinal analysis. Personality And Social Psychology Bulletin, 31(3), 368378. https://doi.org/10.1177/0146167204271597

Paleari, F. G., Regalia, C., \& Fincham, F. D. (2009). Measuring offence-specific forgiveness in marriage: The marital offence-specific forgiveness scale (MOFS). Psychological Assessment, 21(2), 194209. https://doi.org/10.1037/a0016068

Pansera, C., \& La Guardia, J. (2012). The role of sincere amends and perceived partner responsiveness in forgiveness. Personal Relationships, 19(4), 696711. https://doi.org/10.1111/j.1475-6811.2011.01386.x

Patrick, S.,Beckenbach,J.,Sells, J., \&Reardon, R.F.(2013).Anempiricalinvestigationintojustice, grace, andforgiveness: Paths to relationship satisfaction. Family Journal, 21(2), 146153. https://doi.org/10.1177/1066480712466540

Pelucchi, S., Paleari, F. G., Regalia, C., \& Fincham, F. D. (2013). Self-forgiveness in romantic relationships: It matters to both of us. Journal of Family Psychology, 27(4), 541549. https://doi.org/10.1037/a0032897

Pinto, C., \& Barros-Oliveira, J. (2006). Felicidade e perdão: Diferenças por sexo, idade e cultura. Psicologia, Educação e Cultura, 10(2), 353368.

Prager, K., \& Fincham, F. D. (2001). Intimacy in human development and interpersonal relationships [Manuscrito submetido para publicação].

Reed, G. L., \& Enright, R. D. (2006). The effects of forgiveness therapy on depression, anxiety, and posttraumatic stress for women after spousal emotional abuse. Journal of Consulting and Clinical Psychology, 74(5), 920929. https:// doi.org/ 10.1037/0022-006x.74.5.920

Rique, J., \& Camino, C. P. S. (2009). O perdão interpessoal em relação a variáveis psicossocias e demográficas. Psicologia: Reflexão e Crítica, 23(3), 525532. https://doi.org/10.1590/S0102-79722010000300013

Rusbult, C. E., Hannon, P. A., Stocker, S. L., \& Finkel, E. J. (2005). Forgiveness and relational repair. In E. L. Worthington Jr. (Ed.), Handbook of forgiveness (pp. 185205). Routledge.

Schumann, K. (2012). Does love mean never having to say you're sorry? Associations between relationship satisfaction, perceived apology sincerity, and forgiveness. Journal of Social \& Personal Relationships, 29(7), 9971010. https://doi.org/10.1177/0265407512448277

Scorsolini-Comin, F, \& Santos, M. A. (2008). Casamento na medida: Uma revisão integrativa de conceitos e instrumentos de mensuração da satisfação conjugal. In S. R. Pasian, E. T. K. Okino, S. R. Loureiro, \& F. L. Osório (Orgs.), Avaliação de personalidade: Técnicas e contextos diversos (pp. 5567) Vetor.

Sharlin, A. S, Kaslow, F., \& Hammerschmidt, H, (2000). Together through thick and thin: A multinational picture of long-term marriages. The Haworth Clinical Practice.

Sheldon, P., Gilchrist-Petty, E., \& Lessley, J. A. (2014). You did what? The relationship between forgiveness tendency, communication of forgiveness, and relationship satisfaction in married and dating couples. Communication Reports, 27(2), 7890. https://doi.org/10.1080/08934215.2014.902486

Stafford, L., David, P., \& McPherson, S. (2014). Sanctity of marriage and marital quality. Journal of Social and Personal Relationships, 31(1), 5470. https://doi.org/10.1177/0265407513486975

Taysi, E. (2010). Forgiveness in marriage: The role of marital adjustment and attributions. Turk Psikoloji Dergisi, 25(65), 5355. 
Thompson, L. Y. (1998). Women, men, and marital quality. Journal of Family Psychology, 2(1), 95100. https:// psycnet.apa.org/doi/10.1037/h0080478

Thompson, L. Y., Snyder, C., Hoffman, L., Michael, S. T., Rasmussen, H. N., Billings, L. S., Heinze, L., Neufeld, J. E., Shorey, H. S., Roberts, J. C., \& Roberts, D. E. (2005). Dispositional forgiveness of self, others, and situations. Journal of Personality, 73(2), 313360. https://doi.org/10.1111/j.1467-6494.2005.00311.x

Wagner, A., \& Falcke, D. (2001). Satisfação conjugal e transgeracionalidade: uma revisão teórica sobre o tema. Psicologia Clínica, 13(2), 1124.

Waldron, V. R., \& Kelley, D. L. (2008). Communicating forgiveness. Sage.

Ysseldyk, R., \&Wohl, M. A. (2012). I forgive therefore I'm committed: A longitudinal examination of commitment after a romantic relationship transgression. Canadian Journal of Behavioural Science, 44(4), 257263. https:// doi.org/10.1037/a0025463

Ana Isabel Mendes-Teixeira

Psicóloga clínica e da saúde no Serviço de Consulta Psicológica da Faculdade de Psicologia e de Ciências da Educação da Universidade do Porto, Portugal.

E-mail: anateixei.psicologia@gmail.com

(1) https://orcid.org/0000-0001-5988-2552

\section{Cidália Duarte}

Professora auxiliar da Faculdade de Psicologia e de Ciências da Educação da Universidade do Porto, Portugal e membro do Centro de Psicologia da Faculdade de Psicologia e de Ciências da Educação da Universidade do Porto, Portugal.

E-mail: cidalia@fpce.up.pt

(1) https://orcid.org/0000-0002-4655-1460

Endereço para envio de correspondência:

Faculdade de Psicologia e de Ciências da Educação da Universidade do Porto. Rua Alfredo Allen, s/n. CEP: 4200-135. Porto - Portugal.

Recebido 29/05/2018

Aceito 25/04/2019

Received 05/29/2018

Approved 04/25/2019

Recibido 29/05/2018

Aceptado 25/04/2019

Como citar: Mendes-Teixeira, A. I., \& Duarte, C. (2021). Perdão e satisfação conjugal: Uma revisão sistemática. Psicologia: Ciência e Profissão, 41 (n.spe 3), 1-18. https://doi.org/10.1590/1982-3703003200730

How to cite: Mendes-Teixeira, A. I., \& Duarte, C. (2021). Forgiveness and marital satisfaction: A systematic review. Psicologia: Ciência e Profissão, 41 (n.spe 3), 1-18. https://doi.org/10.1590/1982-3703003200730

Cómo citar: Mendes-Teixeira, A. I., \& Duarte, C. (2021). Perdón y satisfacción conyugal: Una revisión sistemática. Psicologia: Ciência e Profissão, 41 (n.spe 3), 1-18. https://doi.org/10.1590/1982-3703003200730 